\title{
Microlensing constraints on the Galactic bulge initial mass function
}

\author{
S. Calchi Novati ${ }^{1,2}$, F. De Luca ${ }^{3}$, Ph. Jetzer ${ }^{3}$, L. Mancini1 ${ }^{1,2}$, and G. Scarpetta ${ }^{1,2}$ \\ 1 Dipartimento di Fisica "E. R. Caianiello", Università di Salerno, via S. Allende, 84081 Baronissi (SA), Italy \\ e-mail: novati@sa.infn.it \\ 2 Istituto Nazionale di Fisica Nucleare, sezione di Napoli, Italy \\ ${ }^{3}$ Institute for Theoretical Physics, University of Zürich, Winterthurerstrasse 190, 8057 Zürich, Switzerland
}

Received 7 August 2007 / Accepted 16 November 2007

\section{ABSTRACT}

\begin{abstract}
Aims. We seek to probe the Galactic bulge IMF starting from microlensing observations.
Methods. We analyse the recent results of the microlensing campaigns carried out towards the Galactic bulge presented by the EROS, MACHO and OGLE collaborations. In particular, we study the duration distribution of the events. We assume a power law initial mass function, $\xi(\mu) \propto \mu^{-\alpha}$, and we study the slope $\alpha$ both in the brown dwarf and in the main sequence ranges. Moreover, we compare the observed and expected optical depth profiles.

Results. The values of the mass function slopes are strongly driven by the observed timescales of the microlensing events. The analysis of the MACHO data set gives, for the main sequence stars, $\alpha=1.7 \pm 0.5$, compatible with the result we obtain with the EROS and OGLE data sets, and a similar, though less constrained slope for brown dwarfs. The lack of short duration events in both EROS and OGLE data sets, on the other hand, only allows the determination of an upper limit in this range of masses, making the overall result less robust. The optical depth analysis gives a very good agreement between the observed and the expected values, and we show that the available data do not allow one to discriminate between different bulge models.
\end{abstract}

Key words. gravitational lensing - Galaxy: bulge - Galaxy: stellar contents

\section{Introduction}

Gravitational microlensing is an established tool for the study and the characterisation of faint compact objects located between the observer and the source stars. It was originally proposed as a tool for the detection of dark matter in the form of MACHOs (Paczyński 1986). Searches towards the Magellanic Clouds by the MACHO (Alcock et al. 2000) and the EROS groups (Tisserand et al. 2007) have placed strong constraints on the possible contribution of a MACHO population to the dark matter halo (for a discussion see, e.g., Mancini et al. 2004; Calchi Novati et al. 2006). A few results have also been obtained with observational campaigns towards M31 by the POINTAGAPE (Calchi Novati et al. 2005) and the MEGA (de Jong et al. 2006) collaborations. On the other hand, the Galactic bulge soon proved to be an almost as interesting target, if not more (Paczynski 1991; Kiraga \& Paczynski 1994) and indeed, by now, the number of observed microlensing events along this line of sight is by two orders of magnitude larger than those observed towards the Magellanic Clouds and M 31. In this case, any contribution from a dark matter MACHO population is expected to be extremely small compared to that of either bulge or disc stars (Griest et al. 1991), so that these studies in principle allow us to constrain the inner Galactic structure. In particular, microlensing observations in this direction have been very important for the assessment of the Galactic triaxial, bar-like, structure (Paczynski et al. 1994; Zhao et al. 1995, 1996; Zhao \& Mao 1996; Bissantz et al. 1997; Gyuk 1999).

Recently, the MACHO (Popowski et al. 2005), OGLE (Sumi et al. 2006) and EROS (Hamadache et al. 2006) collaborations presented the results out of their several-year campaigns towards the Galactic bulge. A remarkable result is the agreement among the different collaborations for the optical depth, in accord with theoretical expectations (Evans \& Belokurov 2002; Bissantz \& Gerhard 2002; Han \& Gould 2003).

While the determination of the optical depth allows the study of the density distribution of the lenses, a more detailed analysis of the shape of the microlensing lightcurves carries much information on the parameters of the lenses. Of particular relevance is the possibility of studying the mass spectrum of the lenses. This approach is based on the relationship between the observed event duration, the Einstein time, and the mass of the lens $t_{\mathrm{E}} \propto \sqrt{\mu_{1}}$. Even if the exact analytical formula shows a dependence also on other unknown physical parameters such as the distances of both lens and source, the relative velocity between them as well as the configuration of the particular lens event, a few conclusions are made possible by the rather large set of observed events at our disposal together with a few reasonable assumptions on the space and velocity distributions of both lenses and sources. A key step to reach the aforementioned agreement of the optical depth between theory and experiments has been the acknowledgment of the severe blending problem resulting in the choice of restricting the sample of source stars to the red clump giant subset (Popowski 2001). In turn, this is essential in the framework of a mass spectrum analysis because of the bias introduced in the evaluation of the Einstein time for blended events.

The determination of the mass function using the results of microlensing searches has been addressed by several 
authors. Han \& Gould (1996) consider a sample of MACHO and OGLE events. Through a likelihood analysis they determine the slope of a power law mass function to be 2.1 in the mass range (0.04-10) $M_{\odot}$. Jetzer (1994) and Grenacher et al. (1999) use the mass moments method to place constraints on the lens masses. In particular, starting from a sample of $41 \mathrm{MACHO}$ events and assuming a Salpeter profile in the mass range $(1-10) M_{\odot}$, Grenacher et al. (1999) constrain the mass function minimum mass and slope below $1 M_{\odot}$, finding $0.012 M_{\odot}$ and 2.0 respectively. Overall, therefore, there is an agreement in attributing a rather large fraction of events to the brown dwarf lens population. On the other hand, Peale (1998) finds no compelling evidence for such a contribution, and evaluates the slope for a power law mass function in the mass range $(0.08-2) M_{\odot}$ to be in the range 2.2-2.5. All of these analyses, we recall, used the complete sample of detected events, not restricted to those with red clump giant sources. Bissantz et al. (2004), considering only red clump giant sources, find a good agreement with the MACHO observed timescale using a mass function with a large contribution from the brown dwarf population (with a power law slope 2.35 in the mass range (0.04-0.35) $\left.M_{\odot}\right)$. Wood \& Mao (2005) extend the Zoccali et al. (2000) slope 1.3 down to a minimum mass of $0.03 M_{\odot}$ compared with the OGLE observed timescale.

In the present paper our aim is to make use of the most recent observational results towards the Galactic bulge to study the mass spectrum of the bulge lens population. The structure of the paper is as follows. Section 2 is devoted to the description of the models we use. In Sect. 3 we point out a few particular features of the usual microlensing quantities upon which we base our method of analysis. In Sect. 4 we present and discuss our main results. In Sect. 5 we conclude.

\section{Models}

In this section we introduce, describe the features, and fix the parameters of our "fiducial" model for the bulge and the disc needed to evaluate the microlensing quantities that we use in the analysis. Furthermore, we discuss a series of changes in the more critical parameters that we use to test the robustness of our results.

\subsection{Density distributions}

\subsubsection{The bulge}

It is now acknowledged that the Galactic bulge has a box-like (tri-axial) structure. In an analysis of clump giant stars, Stanek et al. (1997) explored several analytical distributions to describe the bulge. As a fiducial model we use their model E2, which gives the best agreement with the observational data, where $\rho(r)=\rho_{0} \exp (-r)$, with $^{1} r=\sqrt{\left(x / x_{0}\right)^{2}+\left(y / y_{0}\right)^{2}+\left(z / z_{0}\right)^{2}}$, $x_{0}=890 \mathrm{pc}$ and axis ratio values $x_{0}: y_{0}: z_{0}=10: 4.3: 2.8$, an inclination angle of the bulge major axis with respect to the line of sight of $\alpha=23.8^{\circ}$ (the bulge is oriented with its longer axis pointing towards us for positive longitude values). More recently Rattenbury et al. (2007b) carried out a similar analysis with a much larger sample of stars. As a result, the model E2 is again favoured, with axis ratio values suggesting a more prolate

\footnotetext{
${ }^{1}$ Here, as in Sect. 2.2 where we discuss the velocity distribution, the coordinates $x, y, z$ indicate the principal axes of the component considered, namely, either of the bulge or of the disc.
}

structure, $x_{0}: y_{0}: z_{0}=10: 3.5: 2.6$, and a more restricted range of bulge inclination values is given, $\alpha \sim\left(24^{\circ}-27^{\circ}\right)$. In the analysis we keep using the Stanek et al. (1997) values, and we test our results against those of Rattenbury et al. (2007b). We truncate the bulge at a corotation radius $R_{\mathrm{C}}=3.5 \mathrm{kpc}$ (Bissantz \& Gerhard 2002). The bulge inclination angle value is still the subject of a somewhat lively debate. Values in the range $\alpha \sim\left(10^{\circ}-30^{\circ}\right)$ have been given by several authors, together with different values for the axis ratio (e.g. Dwek et al. 1995; Sevenster et al. 1999; Picaud \& Robin 2004), but recently also much larger values have been suggested. Indeed, Cabrera-Lavers et al. (2007) (and reference therein) discuss a more complicated inner Galactic structure with the co-existence of a double structure, composed of a long $(\sim 4 \mathrm{kpc})$ thin and lighter bar located at low Galactic latitudes, $|b|<2^{\circ}$, and out to high Galactic longitude, with an extremely large value for the inclination angle $\sim 43^{\circ}$, and a distinct triaxial bulge with smaller inclination angle, $\sim 13^{\circ}$. The star count results of GLIMPSE (Benjamin et al. 2005), in the $l=10^{\circ}-30^{\circ}$ range, seem to confirm this result. Such a structure may of course give rise to interesting microlensing signatures, however the currently available data are not suitable for its study, as they exclude the Galactic plane region and are mostly restricted to events observed at small Galactic longitudes. (The EROS collaboration (Tisserand et al. 2007) evaluates a bulge orientation angle of $49^{\circ} \pm 8^{\circ}$ even if they observe only the region out to $|l| \sim 10^{\circ}$ and do not observe any field for $|b|<1^{\circ}$ and only a very few in the band out to $|b| \sim 2^{\circ}$.) The issue of the bulge inclination has also been discussed in Wood (2007) in the framework of an analysis of the microlensing optical depth. As an alternative bulge distribution, Han \& Gould $(1995,2003)$ use the model $\mathrm{G} 2$, favoured by an analysis of the COBE DIRBE observations (Dwek et al. 1995), that they correct for small $(r<700 \mathrm{pc})$ galactocentric distance with the Kent (1992) model. We compare these two models to the observed optical depth. The total bulge mass is usually evaluated in the range $M_{\text {bulge }} \sim(1-2) \times 10^{10} M_{\odot}$ (Blum 1995; Zhao et al. 1996; Dehnen \& Binney 1998). Lacking any compelling constraints we choose to normalise the bulge distribution to the observed value of the microlensing optical depth (Sect. 4.1). Throughout the paper we use $R_{0}=8 \mathrm{kpc}$ as the value for the distance to the Galactic centre.

\subsubsection{The disc}

The profile of the disc distribution is better constrained than that of the bulge, although the value of the parameters that characterise it is subject to debate. In order to parametrise the model we follow closely Han \& Gould (2003) who use a sech ${ }^{2}$ (exponential) profile for the thin (thick) components and normalise the distribution so as to obtain a local stellar density of $\Sigma_{0}=36 M_{\odot} \mathrm{pc}^{-2}$. We note however that Han \& Gould (2003) attribute a rather large density fraction to the "thick" disc, whereas this component is usually reported to contribute only to a minor fraction of the overall density (e.g. Dehnen \& Binney 1998; Vallenari et al. 2006). For our fiducial model we assume, as compared to that of Han \& Gould (2003), the extreme case where we set to zero the thick disc contribution (in their notation, we use $\beta=0$ instead of $\beta=0.565$ ); moreover, we fix the value of the local disc density in agreement with the normalisation of the disc mass function (Sect. 2.3). We have then tested our results using the values of Han \& Gould (2003) and also the Freudenreich (1998) profile characterised by a decreasing density towards the Galactic centre. As already pointed out by Han \& Gould (2003), we find that our results do not depend significantly upon the disc 
model as the bulge component gives by far the dominant contribution to the observed events.

\subsection{Kinematic models}

To evaluate the microlensing rate we have to specify the velocities of the components involved. For bulge and disc stars we take into account both the bulk and random components of motion. For the former, for both disc and bulge we assume a solid body rotation out to $R_{\text {cut }}$ and at outer radii a flat rotation with $R_{\text {cut }}=2(1) \mathrm{kpc}$ and $V_{\max }=220(50) \mathrm{km} \mathrm{s}^{-1}$ for the disc (bulge) component respectively. As the bulge value is less well constrained (Blum 1995; Dehnen 2000; Bissantz et al. 2003; Minchev et al. 2007; Rich et al. 2007), we have tested our results varying the bulge component by $30 \%$ to larger and smaller values. Furthermore, we take into account the solar motion.

For the random component, we assume the velocity distributions to follow an anisotropic Gaussian profile. For the disc dispersion we use $\sigma_{x}=20 \mathrm{~km} \mathrm{~s}^{-1}$ and we consider a linear increase towards the Galactic centre for the remaining components with $\left(\sigma_{y}, \sigma_{z}\right)=(30,20) \mathrm{km} \mathrm{s}^{-1}$ and $\left(\sigma_{y}, \sigma_{z}\right)=(75,50) \mathrm{km} \mathrm{s}^{-1}$ in the local neighbourhood and at the Galactic centre respectively (Han \& Gould 1995). For the bulge, whose velocity dispersions are not as well constrained, we consider two somewhat opposite cases. As a first approach, we follow Han \& Gould (1995) and fix the dispersion values using the virial theorem as applied to the bulge distribution (Blum 1995). For our fiducial model we obtain $\sigma_{x, y, z}=(112.5,86.1,72.1) \mathrm{km} \mathrm{s}^{-1}$. In Sect. 4.2 we investigate the effects of changes with respect to our fiducial model. Whenever we modify either the central density or the pattern speed of the bulge component, we adjust the dispersion values of the bulge according to the prescription of the virial theorem. In only one case we arrive at rather significant differences (beyond a few percent), namely, when we consider as a disc model that of Han \& Gould (2003). Indeed, in that case, our evaluation of the bulge central density decreases by about $25 \%$ (Sect. 4.1 ), implying $\sigma_{x, y, z}=(96.3,74.3,62.8) \mathrm{km} \mathrm{s}^{-1}$.

As a second estimate, we make use of recent observational results (Kozłowski et al. 2006; Rattenbury et al. 2007a) and use $\left(\sigma_{1}, \sigma_{b}\right)=3.0,2.5 \mathrm{mas} \mathrm{yr}^{-1}$, with $\sigma_{\text {los }} \sim 110 \mathrm{~km} \mathrm{~s}^{-1}$ (Binney \& Merrifield 1998). For a bulge inclination of $\alpha=23.8^{\circ}$ and $R_{0}=$ $8 \mathrm{kpc}$ we then evaluate the dispersion along the bulge principal axes to be $(109.4,114.8,94.8) \mathrm{km} \mathrm{s}^{-1}$.

\subsection{Mass functions}

The main aim of the present work is to analyse the microlensing events to place constraints on the mass function of the bulge stars. Zoccali et al. (2000) study the bulge mass function in the range $(0.15-1) M_{\odot}$, finding a good fit to the data with a IMF power law, $\xi(\mu) \propto \mu^{-\alpha}$, with $\alpha=1.3 \pm 0.1$. They also propose a power law with a change of slope at $0.5 M_{\odot}$ and $\alpha \sim 1.4,2.0$ respectively below and above this threshold. Overall, this result is compatible with the previous analysis of Holtzman et al. (1998). The more difficult part of the mass spectrum to be explored is the low mass tail, including very low mass main sequence stars and the brown dwarf range. In their analysis of microlensing events towards the bulge, Han \& Gould (2003) extend the Zoccali et al. (2000) mass function down to well below the hydrogen mass burning limit, at $0.03 M_{\odot}$, and the same was done more recently by Wood \& Mao (2005). Gould (2000) describes how to treat remnants, assuming that all of the stars with mass above $1 M_{\odot}$ have by now entered the remnant phase. Given a slope of the
IMF in this mass range, Gould (2000) proposes $\alpha=2$, it is then possible to evaluate the number and mass fractions due to each of these components (white dwarfs, neutron stars and black holes).

Following the previous results, we assume a power law mass function for both the brown dwarf and the main sequence ranges. We introduce two parameters, the slopes $\alpha_{\mathrm{BD}}, \alpha_{\mathrm{MS}}$ in the mass ranges $(0.01-0.08) M_{\odot},(0.08-1.0) M_{\odot}$ respectively, that we want to constrain. According to analyses carried out for the disc, the slope should change below the hydrogen burning limit (e.g. Kroupa 2007). We also test the effects on our results of two changes on the bulge mass function, namely we introduce a slope change at $0.5 M_{\odot}$, using $\alpha=2$ above this limit (Zoccali et al. 2000), and we move the lower brown dwarf limit from 0.01 to $0.04 M_{\odot}$. We follow Gould (2000) to deal with the remnants, with $(0.6,1.35,5.0) M_{\odot}$ taken as the mass values for white dwarfs, neutron stars and black holes respectively. Besides the value $\alpha_{\text {rem }}=2$, in this mass range we will further test our result with the higher value $\alpha_{\text {rem }}=2.7$, as suggested by disc results. Note that for every pair of values $\left(\alpha_{\mathrm{BD}}, \alpha_{\mathrm{MS}}\right)$, the number and the mass fractions of the various lens components change accordingly.

For the disc mass function we closely follow Kroupa (2002, 2007), with a power law with slopes $0.3,1.3,2.3$ in the mass ranges $((0.01-0.08),(0.08-0.5),(0.5-1.0)) M_{\odot}$ (the low value in the brown dwarf region is in agreement with Allen et al. 2005),

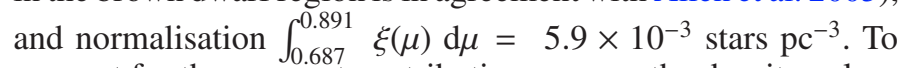
account for the remnant contributions we use the density values reported in Chabrier (2003), to obtain $\Sigma^{\mathrm{rem}}=3 M_{\odot} \mathrm{pc}^{-2}$. This fixes the overall local density for our fiducial disc model to $4.4 \times$ $10^{7} M_{\odot} \mathrm{kpc}^{-3}$.

\section{Analysis: the microlensing quantities}

Our main tool of investigation is the rate of microlensing events $\Gamma$, that carries the information of the number of events per time interval, whereas the microlensing optical depth, $\tau$, is the instantaneous probability of a star being magnified above a given threshold (e.g. Roulet \& Mollerach 1997). Through the analysis of the differential rate, given the efficiency of the experiment, one can analyse the distribution of the relevant microlensing parameters as well as evaluate the number of expected microlensing events.

The microlensing rate (De Rujula et al. 1991; Griest 1991) depends, for both sources and lenses, on the density and velocity distributions, on the lens mass function and on the microlensing configuration. Once the theoretical expression for the differential rate is obtained (Appendix A), to compare with the results of a given experiment, we still need to specify the efficiency of the analysis, usually provided as a function of the microlensing timescale together with the value of the maximum impact parameter allowed.

Throughout the paper we will only consider the simpler microlensing event configuration, point-mass lens and source with uniform relative motion between lens and source, the socalled Paczyński lightcurve (Paczyński 1986). The effects of non-standard configuration events for the evaluation of the microlensing quantities have been the object of a detailed study by Glicenstein (2003). The largest changes are to be expected for binary caustic crossing events, but these represent only a very small fraction of the overall set so that the modifications in the evaluated quantities should not exceed $10 \%$. 

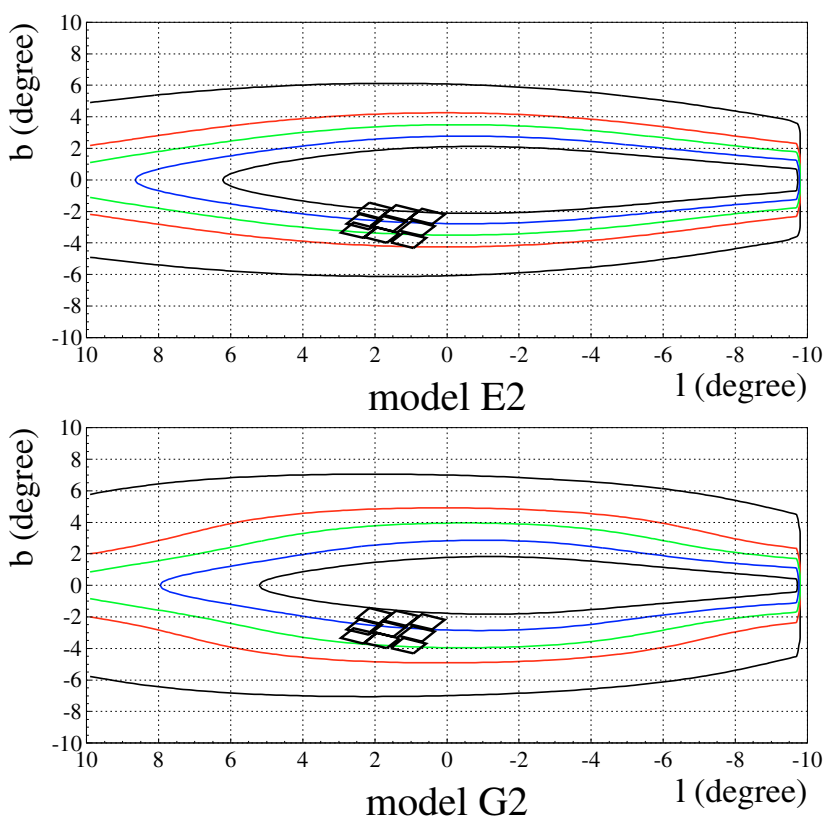

Fig. 1. Optical depth profiles as a function of the Galactic coordinates $l, b$ for the bulge models E2 (top) and G2. The contours of the 9 MACHO CGR fields are shown. The optical depth is normalised to the value of the observed $\tau$ at position $l, b=1.50,-2^{\circ} .68$ (see text for details). The profiles are drawn at values of $\tau=(0.3,1.0,1.5,2.17,3.0) \times$ $10^{-6}$. Overall, the 94 MACHO fields (Popowski et al. 2005) extend in the ranges $0^{\circ}, 8^{\circ}$ and $-2^{\circ},-10^{\circ}$ in Galactic longitude and latitude respectively. The 66 EROS fields (Hamadache et al. 2006) cover two regions at both positive and negative Galactic latitude, $l \sim\left(8^{\circ},-6^{\circ}\right)$, $b \sim\left(-2^{\circ},-6^{\circ}\right)$ and $l \sim\left(6^{\circ},-4^{\circ}\right), b \sim\left(2^{\circ}, 6^{\circ}\right)$. The 30 OGLE fields analysed in Sumi et al. (2006) cover a smaller region near the Galactic centre spreading only slightly beyond the MACHO CGR fields.

\section{Results}

\subsection{The optical depth profiles}

The agreement among the different collaborations (MACHO, EROS and OGLE) on the value of the observed optical depth, and its agreement with theoretical models is, as already noted, a significant result of the microlensing searches towards the Galactic bulge. We take advantage of this result by making the choice to normalise the bulge central density to the observed value of the optical depth. As a fiducial value we take the result reported by the MACHO collaboration towards the "CGR" ("Central Galactic Region", defined in Popowski et al. (2005) as 9 out of the 94 observed fields nearest to the Galactic centre), namely $\tau=2.17_{-0.38}^{+0.47} \times 10^{-6}$ for $(l, b)=1.50,-2.68$. For our fiducial model, the Stanek et al. (1997) model E2, this gives us a central bulge density of $\rho_{0}=9.6 \times 10^{9} M_{\odot} \mathrm{kpc}^{-3}$, corresponding to a bulge mass out to $2.5 \mathrm{kpc}$ of $1.5 \times 10^{10} M_{\odot}$ (this is strictly the mass due to possible lenses). For the model G2 we obtain instead $\rho_{0}=2.4 \times 10^{9} M_{\odot} \mathrm{kpc}^{-3}$ and a mass of $1.4 \times 10^{10} M_{\odot}$.

Having normalised our model to the optical depth observed along a given line of sight, next we have to test the optical depth profile against the observed one, given that the observed events are spread over $\sim 4^{\circ}$ in Galactic latitude and $\sim 10^{\circ}$ in Galactic longitude. Indeed, the optical depth profile depends strongly on the line of sight, in particular on the Galactic latitude (e.g. Evans $\&$ Belokurov 2002). In Fig. 1 we show the optical depth profile for the models E2 and G2. We note the larger gradient along the Galactic latitude for the first model.
To gain further insight on their results, the EROS collaboration (Hamadache et al. 2006) studied the relation $\tau=\tau(b)$ giving the empirical expression $\tau=N \exp \left[-a\left(|b|-3^{\circ}\right)\right]$, where $a, N$ are to be determined from the observational data. For the EROS data set, Hamadache et al. (2006) find $N=1.62 \pm 0.23$, $a=0.43 \pm 0.16$. As a theoretical prediction, given the EROS observational setup, for the E2 (G2) models we find $N, a=$ $1.77,0.52(1.87,0.37)$ respectively. If we carry out the same exercise considering either the MACHO or the OGLE observational setup we find the values $N, a=1.60,0.56(1.72,0.39)$ and $N, a=1.81,0.51(1.94,0.34)$ respectively. Overall, the E2 and the G2 model predictions are both consistent with the observed values.

The previous analysis has to be carried out taking bins in the Galactic latitude, averaging over the Galactic longitude for the observed fields. This way, however, one misses the information of the (albeit smoother) variation of the optical depth profile along the Galactic longitude. Moreover, of course, we are comparing the expected optical depth to the EROS observed values only. As a different approach, we propose to take bins, instead, in the expected optical depth, to be compared with the observed one as evaluated for each observational campaign. To perform this analysis we first need to evaluate the observed value of the optical depth, and therefore the number of sources stars, in each chosen bin, whereas this number is known per field. As a first order approximation we consider the number of source stars in a given fraction of a field to be proportional to its area. A bin in the theoretical optical depth delimits a region in the Galactic plane. We choose the bin sizes so to get a (roughly) equal number of observed events in each bin. For EROS (MACHO) we tried with both 5 and 10 (3 and 5) bins, resulting in very similar results; for OGLE we use 3 bins. In Fig. 2 we show the observed optical depth as a function of the expected one, for the EROS and MACHO and OGLE data sets (with 5, 3 and 3 bins respectively) and both models E2 and G2. We find very good agreement for both models with the three data sets. Indeed, if we fit the relation $\tau_{\mathrm{obs}}=a \cdot \tau_{\mathrm{th}}$, considering the 11 points of the three data sets together, we get $a=0.9 \pm 0.1$, for a reduced $\chi^{2}=1$ for both models.

A possible way to disentangle the different models would come from an independent normalisation of the bulge mass. Indeed, according to our choice, the expected optical depth is made to coincide at the CGR MACHO location, therefore, even if different, the two profiles remain rather near each other along the observed fields. The other option would be to observe events over a larger area of the sky. The more interesting region to be explored being that closer to the Galactic plane.

\subsection{The Galactic bulge IMF}

The microlensing rate, as discussed in Sect. 3, is an efficient tool for the analysis of the characteristics of the microlensing events. Here we focus on an analysis of the timescales provided by the current observations. Indeed, as outlined in the Introduction, though degenerate with other unobservable quantities (distances and relative velocity between sources and lenses) the dependence on the lens mass makes the timescale a valuable source of information on the mass function of the lens population. Due the above-mentioned degeneracy, one needs a rather large number of observed events to deal with them statistically. The current observational results begin to provide such data set, 62 events from the MACHO collaboration (Popowski et al. 2005), 120 events from the EROS collaboration (Hamadache et al. 2006) and the 32 events from the OGLE collaboration (Sumi et al. 2006). Note 

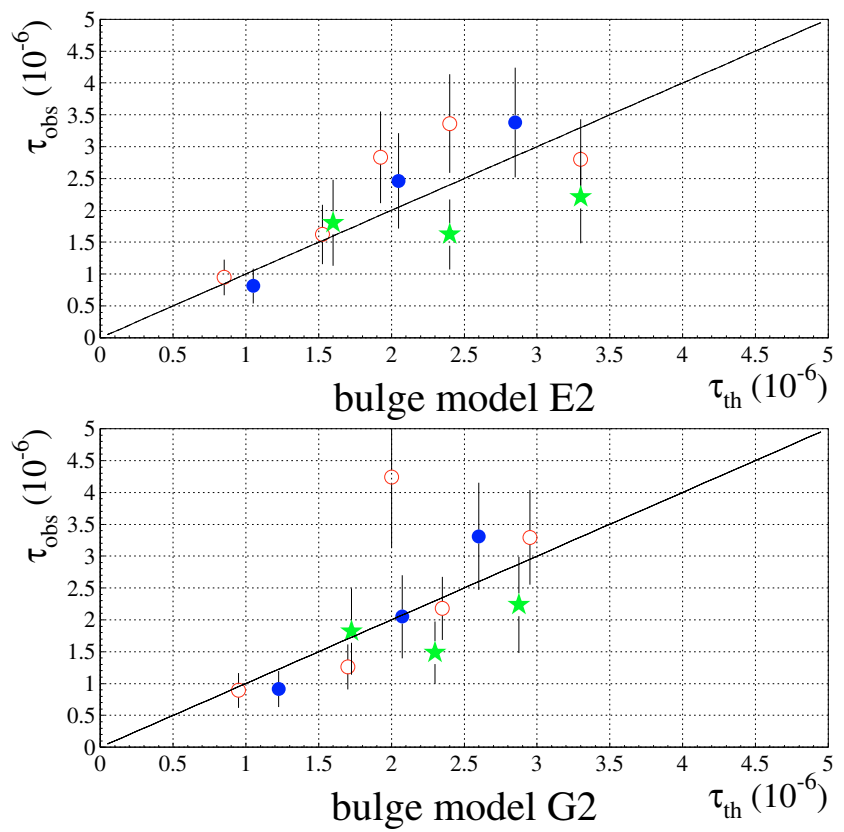

Fig. 2. The observed and the expected optical depth for the bulge models E2 (top) and G2 (see text for details). EROS, MACHO and OGLE data are the empty, filled circles and stars respectively. The solid line is the $y=x$ line.

that we choose not to consider the different data sets together, rather, we carry out independent analyses and then compare the results.

The model, as described in the previous section, together with the microlensing event geometry and the experimental apparatus, summarised in the reported detection efficiency usually given as a function of the duration, $\mathcal{E}=\mathcal{E}\left(t_{\mathrm{E}}\right)$, provides us with the expected number density of the microlensing events. Allowing for the Poisson nature of the process we can write down the likelihood (Gould 2003), as a function of the free parameters of our model, as ${ }^{2}$

$L\left(\alpha_{\mathrm{BD}}, \alpha_{\mathrm{MS}}\right)=\left.\exp \left(-N_{\mathrm{exp}}\right) \prod_{i=1}^{N_{\mathrm{obs}}} \frac{\mathrm{d} \Gamma_{i, \mathcal{E}}}{\mathrm{d} t_{\mathrm{E}}}\right|_{t_{\mathrm{E}, \text { event }}}$.

Here $N_{\text {exp }}$ is the overall expected number of events, to be evaluated by integrating out the differential rate taking into account, besides the detection efficiency, the number of sources and the overall duration of the experiment. In particular it results $N_{\exp }=N_{\exp }\left(\alpha_{\mathrm{BD}}, \alpha_{\mathrm{MS}}\right)$.

As outlined in Sect. 2.3 we take as free parameters the slopes of the IMF in the brown dwarfs and main sequence ranges, $\alpha_{\mathrm{BD}}, \alpha_{\mathrm{MS}}$, that we want to estimate. To evaluate the likelihood, we sum the disc and the bulge contributions, and for each the contribution of the brown dwarfs, main sequence and remnants lens populations.

Finally, to estimate the confidence levels, we evaluate the probability distribution $P\left(\alpha_{\mathrm{BD}}, \alpha_{\mathrm{MS}}\right)$ by Bayesian inversion using a flat prior on both the parameters.

It is useful, for our purposes, to take the sample of MACHO CGR events as a "fiducial" sample. This provides us with a more homogenous, but still quite large, set of events all located in a region small enough to make any possible spatial dependence, that

2 This is the so-called "extended maximum likelihood", first proposed by Fermi (for a discussion see e.g. Barlow 1989), that is appropriate in experiments where the number of events is itself a random variable.
Table 1. The average observed duration, $\left\langle t_{\mathrm{E}}\right\rangle$ (days), of the microlensing candidates reported by the MACHO (Popowski et al. 2005), EROS (Hamadache et al. 2006) and OGLE (Sumi et al. 2006) collaborations. In the first row, for MACHO and EROS we report the result within the CGR (see text for details). For each data set, in the right column we report the average weighted by the inverse efficiency.

\begin{tabular}{c|cc|cc|cc}
\hline \hline & \multicolumn{2}{|c}{ MACHO } & \multicolumn{2}{c}{ EROS } & \multicolumn{2}{c}{ OGLE } \\
\hline CGR & 19.5 & 15.0 & 25.9 & 22.4 & - & - \\
all set & 28.0 & 20.0 & 32.9 & 28.3 & 32.8 & 28.1 \\
\hline
\end{tabular}

we may not have correctly reproduced within our model, almost irrelevant. Furthermore, the CGR allows a more straightforward comparison among the different data sets.

The 66 EROS fields (Hamadache et al. 2006) cover a rather larger region in the plane of the sky than the MACHO fields, both at positive and negative Galactic latitude. For comparison with the MACHO CGR sample, we select the 5 fields $(5,8,607$, $610,611)$ whose location is roughly coincident with that of the MACHO CGR fields, and where 18 of the 120 events reported by the EROS collaboration are located.

Finally, we observe that the location of the 20 fields used by OGLE in their analysis (Sumi et al. 2006) only slighly exceeds the CGR.

For the observed distributions for both the MACHO and the EROS data sets, there is an increase in duration moving from the smaller sample in the CGR to the complete data set. In Table 1 we report the average observed durations, both uncorrected for the efficiency and weighted by the inverse efficiency, the latter quantity allowing a more straightforward comparison between the different data sets.

\subsubsection{The analysis within the CGR}

The main result of the present paper is shown in Fig. 3. From the maximum likelihood analysis we show the contours of equal probability in the $\alpha_{\mathrm{BD}}, \alpha_{\mathrm{MS}}$ parameter space. Here we consider the sample of the 42 MACHO events observed within the CGR.

The data better constrain the IMF slope in the main sequence range than in the brown dwarf range. As for the IMF parameters, at maximum probability we get the values $\alpha_{\mathrm{BD}}=$ 1.6, $\alpha_{\mathrm{MS}}=1.7$. The corresponding bulge mass fractions are $\sim(21 \%, 56 \%, 17 \%, 4 \%, 3 \%)$ for brown dwarfs, main sequence, white dwarfs, neutron stars and black holes, respectively, for an average mass of $0.1 M_{\odot}$. Note the rather high brown dwarf fraction, indeed within the $34 \%$ level it does not decrease below $\sim 20 \%$. Overall the bulge contributes about $80 \%$ of the events (this result confirming the statement made about the only relative importance of the disc contribution, Sect. 2.1.2) and the event fractions due to the different lens populations are $\sim(29 \%, 57 \%, 11 \%, 2 \%, 1 \%)$. In Fig. 4 , we show the one dimensional probability profile $P\left(\alpha_{\mathrm{BD}}\right)$ and $P\left(\alpha_{\mathrm{MS}}\right)$. As already mentioned, the $\alpha_{\mathrm{MS}}$ distribution turns out to be more peaked, with $\alpha_{\mathrm{MS}}=1.7 \pm 0.5$ and $\alpha_{\mathrm{BD}}=1.6 \pm 1.0$.

In Fig. 3 we show the lines of equal value of the expected duration $t_{\mathrm{E}}$ superimposed on the likelihood probability contours. As it is apparent from the plot, the lines of degeneracy in the parameter space $\alpha_{\mathrm{MS}}-\alpha_{\mathrm{BD}}$ that are found in the probability contours are driven by the duration (within the innermost $34 \%$ probability contour the dispersion of the expected duration is only about $5 \%$ ). In particular, we observe that expected shorter durations are associated with steeper mass function. This is expected, of course, because of the relationship between the duration and 


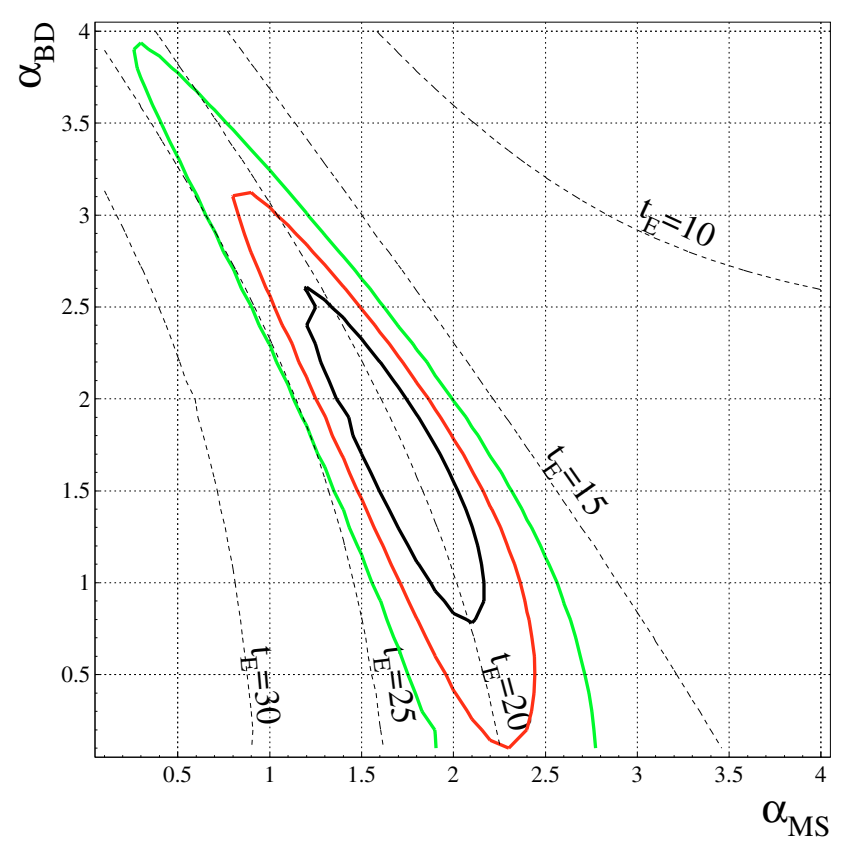

Fig. 3. Probability isocontours with $34 \%, 68 \%$ and $90 \%$ regions in the $\alpha_{\mathrm{BD}}, \alpha_{\mathrm{MS}}$ plane. $\alpha_{\mathrm{BD}}, \alpha_{\mathrm{MS}}$ are the slopes of the power law IMF of the Galactic bulge lenses, in the brown dwarf and main sequence range, respectively. The dashed lines are the lines of equal average expected event durations, for the values of 10, 15, 20, 25 and 30 days. Larger values of the duration are found for smaller values of the IMF slopes. Here the set of 42 events reported by the MACHO collaboration in the CGR is considered.

the mass of the lens. This correlation is relevant in order to properly understand the variations we find in the evaluated slopes of the mass function for either sets of data with different duration distributions or for different models.

We now compare the results we obtain using the sample of MACHO microlensing candidates (Popowski et al. 2005) with that of the EROS (Hamadache et al. 2006) and of the OGLE (Sumi et al. 2006) collaborations. Following the previous discussion, we first consider the samples restricted to the inner Galactic region, namely we use 18 out of the 120 EROS microlensing candidates and the full set of the 32 OGLE microlensing candidates.

As shown in Fig. 5 (top panel), the analysis over the EROS data set allows us to determine the maximum for the IMF slope in the main sequence region, roughly consistent with that found using the MACHO data set, but does not reveal any lower limit in the brown dwarf range. This arises because of the different distribution of the observed timescale. In particular, the explanation may be traced back to the lack (already noted in Hamadache et al. 2006) of very short duration events, say below 5 days, within the EROS data set (both in the restricted sample of 18 events we consider here and in the full data set). While this difference does not significantly affect the results on the optical depth, in the present analysis this turns out to be very relevant. The analysis performed on the OGLE data set provides a qualitatively similar result. In agreement with the previous discussion, we note that the somewhat lower value for $\alpha_{\mathrm{MS}}$ is a consequence of the higher average observed timescale.

The above analysis clearly shows the extent to which short duration events are essential to constrain the lower tail of the IMF. We further address this question, also to compare the results we obtain with the different data sets, by mean of the following analysis. We set to zero the efficiency below a given
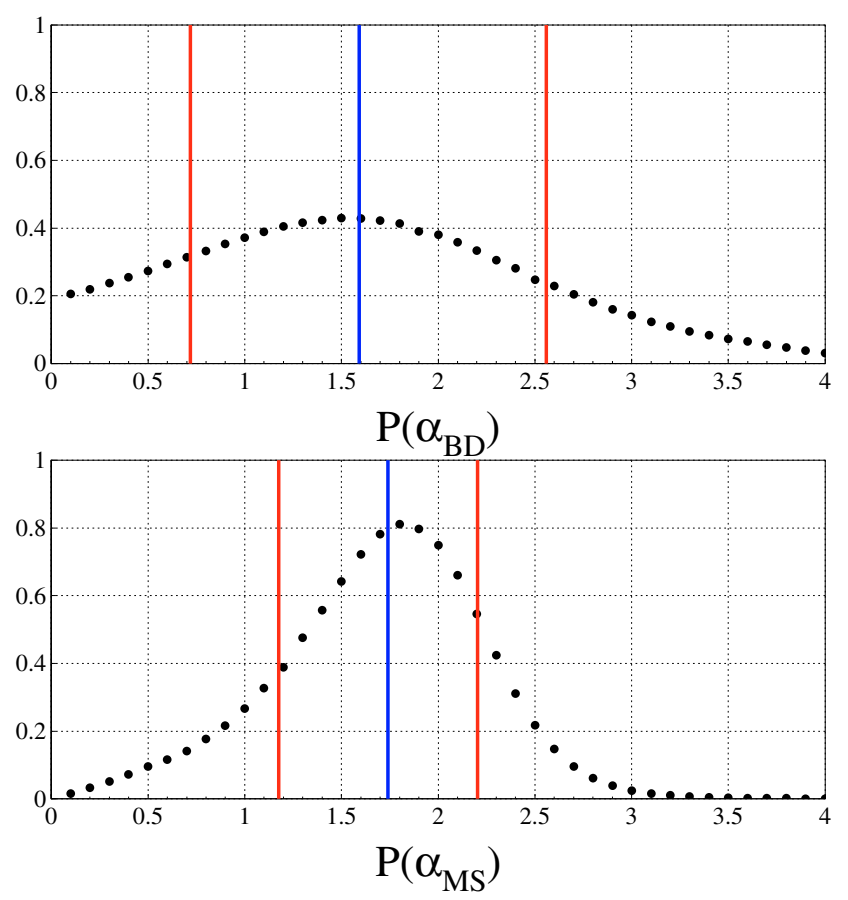

Fig. 4. Probability distribution for the Galactic bulge power law IMF slopes in the brown dwarf (top) and main sequence mass ranges. Here the set of 42 events reported by the MACHO collaboration in the CGR is considered. The probability lines of $16 \%, 50 \%$ and $84 \%$ are indicated.

threshold, in particular $\mathcal{E}\left(t_{\mathrm{E}}<5 \mathrm{~d}\right)=0$ and at the same time we exclude from the analysis those observed events with $t_{\mathrm{E}}<5 \mathrm{~d}$, namely, the 6 events from the MACHO sample.

The likelihood contours we obtain for the MACHO data set are shown in the bottom panel of Fig. 5. Comparing with Fig. 4 we see that, as for the EROS and the OGLE data sets, the brown dwarf slope is no longer bounded at its lower end, while the main sequence one peaks roughly in the same region. Carrying out this analysis for the EROS data set we find an almost identical result to that shown in the top panel of Fig. 5, while for OGLE we find a somewhat different behaviour. In that case a lower bound for $\alpha_{\mathrm{BD}}$ appears, at least for the innermost 34\% contour, but at the same time the contours become unbounded at the upper end. A similiar behaviour is also observed when we move the lower limit on the lens mass from 0.01 to $0.04 M_{\odot}$, Sect. 4.2.3, and this can be understood, as we are excluding the duration range where the microlensing rate of very large brown dwarf slopes peak.

\subsubsection{The analysis of the complete data set}

The analysis of the complete data sets confirm our previous conclusions. In Fig. 6 we show the probability contours for both the full set of events of MACHO and EROS (for OGLE, the results obtained with the full data set are shown in Fig. 5). For the MACHO data set we evaluate the slope in the main sequence range to be $\alpha_{\mathrm{MS}}=1.6 \pm 0.4$, in agreement with the previous result. With respect to Figs. 3 and 5 we observe, however, the maximum likelihood contours moving towards somewhat smaller values of the IMF slopes. This is of course to be attributed to the increase in the observed duration (Table 1). Comparing to Fig. 5, for both MACHO and EROS data sets we observe a shrinking in the probability contours due to the much larger sample of events used in the present analysis. 

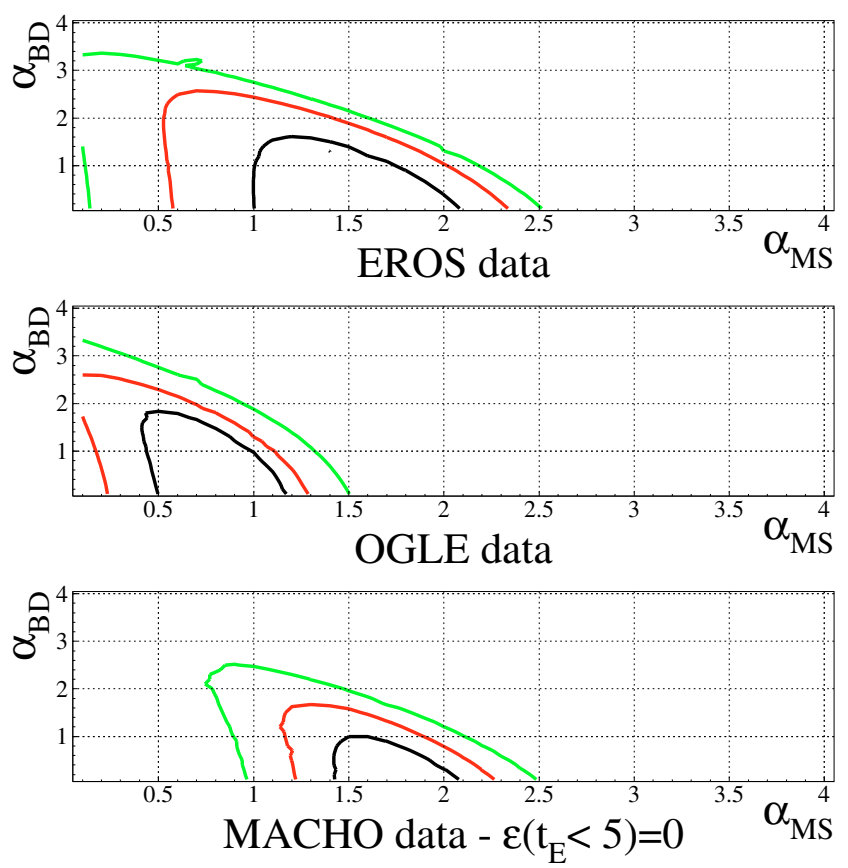

Fig. 5. Probability isocontours with $34 \%, 68 \%$ and $90 \%$ regions in the $\alpha_{\mathrm{BD}}, \alpha_{\mathrm{MS}}$ plane. $\alpha_{\mathrm{BD}}, \alpha_{\mathrm{MS}}$ are the slopes of the power law IMF of the Galactic bulge lenses, in the brown dwarf and main sequence ranges, respectively. From top to bottom, the results of the analysis for the EROS and OGLE data and the results of an analysis of the MACHO data where we set the efficiency below $t_{\mathrm{E}}<5 \mathrm{~d}$ to zero (see text for details). For the MACHO and EROS data sets we restrict the analysis to the subset of events observed in the inner Galactic region.

\subsubsection{The IMF: a test against the fiducial model}

We now focus on the possible systematic effects resulting from a change in the characteristics of our fiducial model (Sect. 2). We carry out this analysis using the MACHO data set only within the CGR. To give an indication of the goodness of the model we use a Kolmogorov-Smirnov (KS) test comparing the expected to the observed duration distribution (we report its significance level, $k s$, as a disproof of the null hypothesis that the distribution are the same, such that a low value of $k s$ indicates a poor agreement between the expected and the observed distribution). Such an analysis makes sense because the variations in the expected timescale do not exceed $\sim 5 \%$ across the CGR. This allows us to carry out the KS test by evaluating an average rate, summing the rate observed towards the different fields with a weight given by the number of source stars that we compare to the observed duration distribution. As test models we consider the following (Table 2): model 2: we change the bulge dispersion velocity to the Rattenbury et al. (2007a) values (Sect. 2.2); model 3-4: we change respectively downward and upward the bulk rotation velocity of the bulge (Sect. 2.2); model 5: we change the parameters of the disc density profile according to Han \& Gould (2003) (Sect. 2.1.2); model 6: we change the disc density profile according to Freudenreich (1998) (Sect. 2.1.2); model 7: we change the bulge scale lengths according to Rattenbury et al. (2007b) (Sect. 2.1.1); model 8: we change the bulge IMF slope to $\alpha_{\text {rem }}=2.7$ (Sect. 2.3); model 9: we change the bulge mass function introducing a second slope in the main sequence range (Sect. 2.3); 10) we change the mass lower limit in the brown dwarf range to $0.04 M_{\odot}$ (Sect. 2.3). In Table 2 we report the results: for each model we give the evaluated $\alpha_{\mathrm{MS}}$ parameter out
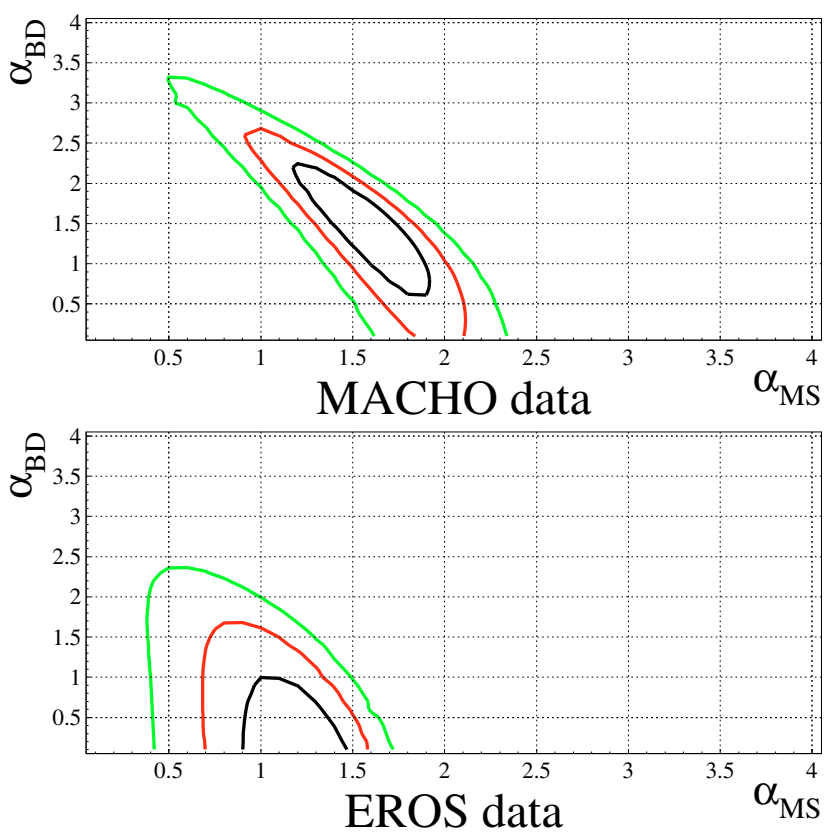

Fig. 6. Probability isocontours with $34 \%, 68 \%$ and $90 \%$ regions in the $\alpha_{\mathrm{BD}}, \alpha_{\mathrm{MS}}$ plane. $\alpha_{\mathrm{BD}}, \alpha_{\mathrm{MS}}$ are the slopes of the power law IMF of the Galactic bulge lenses, in the brown dwarf and main sequence ranges, respectively. The full set of events for MACHO (top) and EROS data sets are considered.

Table 2. Results of the maximum likelihood analysis on the MACHO data set (Popowski et al. 2005) for our fiducial model and for the different models discussed (Sect. 2). $\alpha_{\mathrm{MS}}$ is the slope of the power law IMF in the main sequence range. $k s$ is the Kolmogorov-Smirnov significance level for the null hypothesis that the expected and observed distributions are the same.

\begin{tabular}{|c|c|c|c|c|c|}
\hline \multirow[t]{2}{*}{ Model } & \multicolumn{3}{|c|}{$\alpha_{\mathrm{MS}}$} & \multirow[t]{2}{*}{$k s$} & \multirow[t]{2}{*}{ Model change } \\
\hline & $16 \%$ & $50 \%$ & $84 \%$ & & \\
\hline 1 & 1.18 & 1.74 & 2.20 & 0.39 & fiducial model \\
\hline 2 & 0.86 & 1.40 & 1.80 & 0.29 & bulge velocity \\
\hline 3 & 1.16 & 1.73 & 2.19 & 0.39 & bulge velocity \\
\hline 4 & 1.17 & 1.74 & 2.20 & 0.39 & bulge velocity \\
\hline 5 & 1.32 & 2.10 & 2.90 & 0.41 & disc model \\
\hline 6 & 1.13 & 1.67 & 2.10 & 0.40 & disc model \\
\hline 7 & 0.97 & 1.51 & 1.92 & 0.33 & bulge model \\
\hline 8 & 0.95 & 1.56 & 2.06 & 0.40 & mass function \\
\hline 9 & 0.90 & 1.62 & 2.21 & 0.40 & mass function \\
\hline 10 & 1.86 & 2.19 & 2.60 & 0.26 & mass function \\
\hline
\end{tabular}

of the $P\left(\alpha_{\mathrm{MS}}\right)$ distribution (with the $16 \%, 50 \%$ and $84 \%$ bound) and the KS significance level.

For the different models the likelihood maximum moves on the $\alpha_{\mathrm{BD}}-\alpha_{\mathrm{MS}}$ plane so as to always peak around the same expected timescale, with the resulting mass function slopes changing accordingly. The largest variation downward, $\alpha_{\mathrm{MS}} \sim 1.4$, is found for model 2 as an effect of the increased bulge velocity dispersions. Note the large value we obtain for model 5 , we find $\alpha_{\mathrm{MS}} \sim 2.1$. Here two different effects push in the same direction towards a steeper mass function, namely a smaller bulge contribution and a decrease in the bulge dispersion velocity. The qualitative shape of the likelihood contours does not change for any of the models except the last. Here, as an effect of the increase of the minimum mass value in the brown dwarf range, from 0.01 to $0.04 M_{\odot}$, the probability distribution for $\alpha_{\mathrm{BD}}$ becomes unbounded at its upper end. Correspondingly, we also find a steeper mass function and lower KS significance level. 


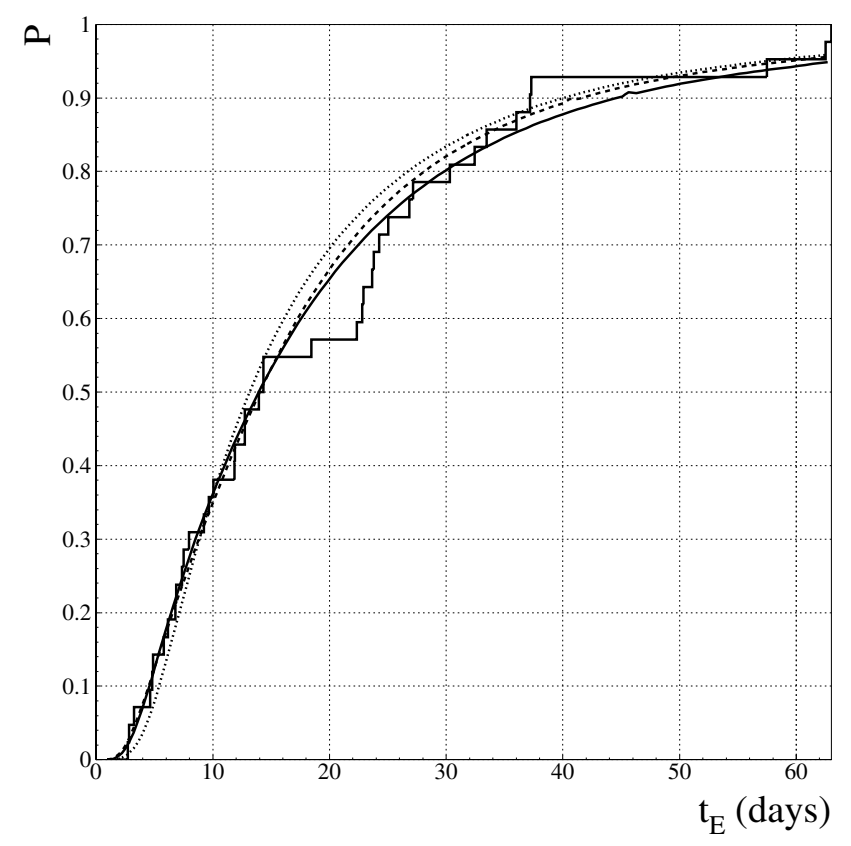

Fig. 7. Cumulative duration distributions $(P)$ for the observed events, together with the the theoretical prediction for three different models. Solid, dashed and dotted lines are for the fiducial model and models 2 and 10, respectively (see text for details). Here the set of 42 events reported by the MACHO collaboration in the CGR is considered.

Overall, the variations we find for $\alpha_{\mathrm{MS}}$ for the different models we have tested do not exceed the statistic uncertainty we have in our fiducial configuration. This is in agreement with the KS analysis, according to which we obtain acceptable results for all the models we consider.

In Fig. 7 we show the cumulative distribution for the sample of the 42 MACHO CGR events together with the theoretical cumulative distributions for the fiducial model and models 2 and 10 , for which we obtain the smallest and the largest values for the main sequence slope ( $\alpha_{\mathrm{MS}}=1.4,2.2$, respectively) and the worst agreement according to the KS test. Besides the lack of observed events at $t_{\mathrm{E}} \sim 20 \mathrm{~d}$, we note in particular the very good agreement with short duration events for both the fiducial model and model 2 and the better agreement with long duration events for model 10.

\subsection{The expected number of microlensing events}

Besides the study of the duration distribution, the analysis of the microlensing rate allows one to evaluate the number of expected events. Through the analysis we have normalised the bulge central density, once having fixed that of the disc, by using the observed value of the optical depth. Because of the relationship between the optical depth and the microlensing rate, through the event duration, we may therefore expect to find a good agreement between the observed and the expected number of microlensing events. Indeed, even if the number of expected events varies by almost a factor of 3 across the $\alpha_{\mathrm{BD}}-\alpha_{\mathrm{MS}}$ parameter space we explore, we find a fair agreement. For the MACHO data set our prediction is compatible within $1 \sigma$ to the observed value; we find 38 and 54 events compared with 42 and 62 events, in the CGR and the complete data set, respectively. For the EROS and OGLE data sets we arrive at an even better agreement, with an expected number of 118 (31) compared with 120 (32). These figures do not vary significantly (at most by $\sim 2$ events) within the innermost $34 \%$ probability contour.

\subsection{The blending issue}

In very crowded fields, such as those observed towards the Galactic centre, the observed objects can be the blend of several stars. This blending effect is a major source of concern for the interpretation of microlensing searches. This is the reason, we recall, that led, in evaluating the optical depth, to the choice of considering only bright sources for which one expects the blending effects to be alleviated. The multiple effects of blending are supposed to roughly balance each other when evaluating the optical depth (see e.g. Hamadache et al. 2006). On the other hand, as blending is expected to cause an underestimation of the evaluated event duration, we may question its relevance with respect to our results.

The extent to which blending contaminates the sample of red clump giants is a subject of debate (Popowski et al. 2005; Sumi et al. 2006; Hamadache et al. 2006; Smith et al. 2007). For the present analysis we remark that both MACHO and EROS evaluate the optical depth without including the effect of blending, while OGLE, who find this effect to be relevant within their data set, use blended fits. Throughout our analysis we have used the reported values of the duration according to this choice.

Popowski et al. (2005), for the MACHO collaboration, identify an "extremely conservative" subset of events for which they evaluate the blend fraction to be very close to 1 . We carry out our analysis on this subsample, composed of 22 events within the CGR. As in Popowski et al. (2005) we introduce an overall normalisation factor for the microlensing rate equal to the ratio of the number of events in this restricted sample to that of the complete sample. This is coherent with the purpose of the analysis, where one wants to test whether blending substantially affects the event parameters, and the derived quantities such as the optical depth and the microlensing rate, while assuming that it does not change the number of detected events. As a result we find somewhat broader contours, because of the smaller number of events, with the brown dwarf slope unbounded at its lower tail, but otherwise fully compatible with our previous results. This is in agreement with our previous discussion. Indeed, the average observed duration for this sample turns out to be similar to the full CGR sample $\left\langle t_{\mathrm{E}}\right\rangle=22.3 \mathrm{~d}$ but 5 out of the 6 very short duration events are excluded.

On the other hand Sumi et al. (2006), for the OGLE collaboration, worked the other way round. They repeated their analysis assuming no blending, finding a new sample of 48 microlensing candidates, with an average duration roughly $20 \%$ shorter than in the 32 events sample. Within this new sample there are 3 candidates with $t_{\mathrm{E}}<2 \mathrm{~d}$, and this is of course relevant in the view of our previous discussion. However, these candidates are strongly affected by blending. We prefer, therefore, not to include them in our analysis. Our likelihood analysis carried out on this 45-event subsample turns is compatible with the previous one.

Finally, we recall that EROS (Hamadache et al. 2006) conclude that blending does not affect significantly their results, and comment the apparent discrepancy with the result obtained by OGLE on this issue on the basis of their different choice for the threshold value of the amplification (in particular, OGLE consider also less amplified events with respect to both EROS and MACHO analyses).

In conclusion, given the available data sets, blending, though relevant, does not appear to significantly affect our results. 

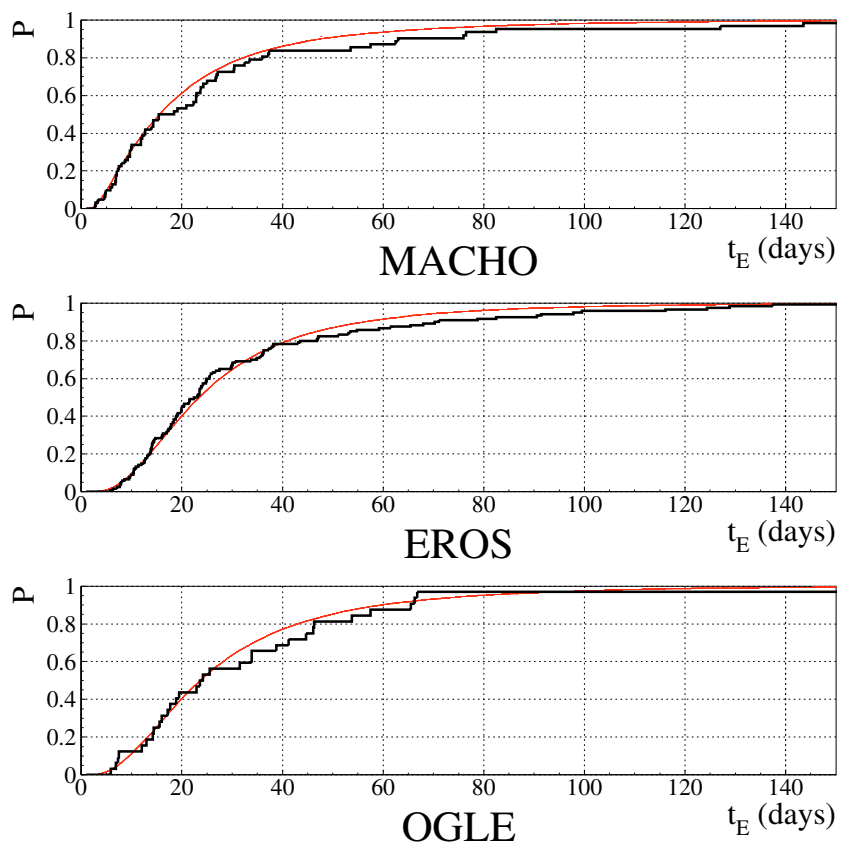

Fig. 8. Cumulative duration distributions $(P)$ for (top to bottom) the MACHO, EROS and OGLE sample of events. Superimposed, the predicted distribution for the fiducial model.

\subsection{Long duration events}

In Fig. 8 we show, for the three complete sets of events we consider (MACHO, EROS and OGLE), the cumulative duration distribution and the expected cumulative distribution for the fiducial model (averaged as for the KS analysis in Sect. 4.2.3), evaluated at the IMF slopes that maximise the likelihood. As for the smaller CGR sample in Fig. 7, we note the rather good agreement especially for short duration events. For both EROS and MACHO data sets we also observe a systematic excess of long duration events. This turns out to be, however, only marginally significant. For instance, the models predict $10 \%$ of events with $t_{\mathrm{E}}>51 \mathrm{~d}$, compared with $\sim 15 \%$ of the observed events.

\section{Conclusions}

We have considered the sample of microlensing events observed towards the Galactic bulge with red clump giant sources reported by the the MACHO (Popowski et al. 2005), OGLE (Sumi et al. 2006) and EROS (Hamadache et al. 2006) collaborations to place constraints on the bulge mass function. In particular, through a likelihood analysis, we have studied the slopes, $\alpha_{\mathrm{BD}}, \alpha_{\mathrm{MS}}$, of a power law mass function in the brown dwarf (0.01-0.08) $M_{\odot}$ and main sequence $\left(0.08-1\right.$.) $M_{\odot}$ mass ranges.

For our fiducial model, comparing to the CGR sample of $42 \mathrm{MACHO}$ events, we obtain $\alpha_{\mathrm{MS}}=1.7 \pm 0.5$. This result compares well to that obtained in the $(0.15-1) M_{\odot}$ range by Zoccali et al. (2000), $\alpha \sim 1.3$. The slope in the brown dwarf range turns out to be less well constrained, $\alpha_{\mathrm{BD}}=1.6 \pm 1$. Overall our maximum likelihood results indicate a rather significant contribution of low mass lenses, with $\sim 30 \%$ of the events to be attributed to brown dwarfs. The last result is in agreement with previous analyses (Han \& Gould 1996; Grenacher et al. 1999), while our value for the main sequence slope, somewhat smaller, may be explained because we are using a more suitable sample of redclump-source events.
The analyses of the EROS and OGLE data sets give us somewhat different results. We derive a smaller value for the slope in the main sequence range, although compatible with the MACHO data set result, but we obtain only an upper limit for the slope of the brown dwarf population. This behaviour finds its explanation in the different observed timescales. In particular, very short timescale events $\left(t_{\mathrm{E}}<5 \mathrm{~d}\right)$, only observed in the MACHO data set, are essential to constrain the brown dwarf mass function. The lack of short timescale events has been noticed in the EROS analysis (Hamadache et al. 2006). In all of the experiments, the detection efficiency of short durations events is extremely low, rendering the analysis in the brown dwarf regime difficult and, therefore, making the result less robust.

More reliable constraints on the mass function may come from a better understanding of the bulge model, but especially, as already stressed, by improving the statistics of observed short duration events.

Furthermore, we have carried out an analysis on the optical depth. The agreement with the expected values is recognized (Han \& Gould 2003). Here we have considered the profile of the expected optical depth as compared to the observed one, finding a good agreement for both the models we have considered, the model E2 of Stanek et al. (1997) and the model G2 of Dwek et al. (1995). To further constrain the bulge profile it would be useful to extend microlensing searches to cover a larger area in the sky plane.

Acknowledgements. We are grateful to the referee, A. Gould, for useful comments and suggestions. We thank K. Griest, C. Hamadache, A. Milsztajn and T. Sumi of the MACHO, EROS and OGLE collaborations for providing the efficiency tables and other data in electronic format. S.C.N. thanks Jean Kaplan for valuable discussions. S.C.N., L.M. and G.S. acknowledge support for this work by MIUR through PRIN 2006 Prot. 2006023491_003 and by research funds of the Salerno University. F.D.L. work was performed under the auspices of the EU, which has provided financial support to the "Dottorato di Ricerca Internazionale in Fisica della Gravitazione ed Astrofisica" of Salerno University, through "Fondo Sociale Europeo, Misura III.4". F.D.L. acknowledges the Forschungskredit of the University of Zürich for financial support.

\section{Appendix A: Evaluation of the microlensing rate}

In this appendix we detail the evaluation of the differential microlensing rate (Sect. 3). This quantity is directly related to the number of expected microlensing events evaluated as

$\mathrm{d} N_{\mathrm{ev}}=N_{\mathrm{obs}} T_{\mathrm{obs}} \mathrm{d} \Gamma$

where $\mathrm{d} \Gamma$ is the differential rate at which a single star is microlensed, $N_{\text {obs }}$ is the number of monitored sources and $T_{\text {obs }}$ is the whole observation time.

The microlensing rate expresses the number of lenses that pass through the microlensing tube $\mathrm{d}^{3} x$ in the time interval $\mathrm{d} t$, for a given number density distribution $n_{1}(\boldsymbol{x})$ and velocity distribution $f\left(\tilde{\boldsymbol{v}}_{1}\right)$ of the lenses. It reads (De Rujula et al. 1991; Griest 1991)

$\mathrm{d} \Gamma=\frac{n_{1}(\boldsymbol{x}) \mathrm{d}^{3} x}{\mathrm{~d} t} \times f\left(\tilde{\boldsymbol{v}}_{1}\right) \mathrm{d}^{3} \tilde{v}_{1} \times \frac{\rho_{\mathrm{s}} D_{\mathrm{s}}^{2-\gamma} \mathrm{d} D_{\mathrm{s}}}{I_{\mathrm{s}}} \times f\left(\tilde{\boldsymbol{v}}_{\mathrm{s}}\right) \mathrm{d}^{3} \tilde{v}_{\mathrm{s}}$

The two last terms account respectively for the spatial and velocity distribution of the sources, with $I_{\mathrm{s}}=\int \rho_{\mathrm{s}} D_{\mathrm{s}}^{2-\gamma} \mathrm{d} D_{\mathrm{s}}$. We take into account that the volume element varies with distance as $D_{\mathrm{s}}^{2} \mathrm{~d} D_{\mathrm{s}}, D_{\mathrm{s}}$ being the distance between observer and source, and that the fraction of monitored stars having a luminosity higher than a minimum detectable luminosity, $L_{*}$, scales as $L_{*}^{-\gamma / 2} \sim D_{\mathrm{S}}^{-\gamma}$ 
(Kiraga \& Paczynski 1994). Throughout the analysis of this paper we use $\gamma=0$, characteristic for bright stars that can be considered, at least approximately, as standard candles.

The volume element of the microlensing tube is $\mathrm{d}^{3} x=$ $\left(\boldsymbol{v}_{\mathrm{r}, \perp} \cdot \hat{\boldsymbol{n}}\right) \mathrm{d} t \mathrm{~d} S . \mathrm{d} S=\mathrm{d} l \mathrm{~d} D_{1}$ is the portion of the tube external surface, $D_{1}$ is the distance between observer and lens, and $\mathrm{d} l=u_{\mathrm{t}} R_{\mathrm{E}} \mathrm{d} \alpha$, where $R_{\mathrm{E}}$ is the lens Einstein radius, $u_{\mathrm{t}}$ is the maximum impact parameter, $\boldsymbol{v}_{\mathrm{r}, \perp}$ is the component of lens velocity in the plane orthogonal to the line of sight (hereafter los), and $\hat{\boldsymbol{n}}$ is the unit vector normal to the tube inner surface at the point where the microlensing tube is crossed by the lens. In the following $\theta$ is the angle between $\boldsymbol{v}_{\mathrm{r}}$ and $\hat{n}$, with $\theta \in\left(-\frac{\pi}{2}, \frac{\pi}{2}\right)$ (one considers only lenses that enter the tube).

The velocity of the lenses entering the tube reads

$\tilde{\boldsymbol{v}}_{\mathrm{l}}=\boldsymbol{v}_{\mathrm{r}}+\boldsymbol{v}_{\mathrm{t}}$,

where $\boldsymbol{v}_{\mathrm{t}}$ is the tube velocity. On the lens plane, we have $\boldsymbol{v}_{\mathrm{t}}=(1-$ $x) \boldsymbol{v}_{\mathrm{obs}}+x \tilde{\boldsymbol{v}}_{\mathrm{s}}$, where $x \equiv \frac{D_{1}}{D_{\mathrm{s}}}, \boldsymbol{v}_{\mathrm{obs}}$ is the observer's (solar) velocity and $\tilde{\boldsymbol{v}}_{\mathrm{s}}$ is the source velocity. We decompose both lens and source velocities into a random plus a bulk component $\tilde{\boldsymbol{v}}=\boldsymbol{v}+\boldsymbol{v}_{\mathrm{drift}}$. In conclusion

$\boldsymbol{v}_{\mathrm{l}}=\boldsymbol{v}_{\mathrm{r}}+x \boldsymbol{v}_{\mathrm{s}}+\boldsymbol{A}$,

where we have defined the vector $\boldsymbol{A}$ so as to include all the bulk motion, $\boldsymbol{A} \equiv\left(\boldsymbol{v}_{\mathrm{obs}}-\boldsymbol{v}_{\mathrm{drift}, 1}\right)+x\left(\boldsymbol{v}_{\mathrm{drift}, \mathrm{s}}-\boldsymbol{v}_{\mathrm{obs}}\right)$. For the random component we use an anisotropic Gaussian distribution (Sect. 2.2).

Looking at Eq. (A.2) we see that we start from the joint three-dimensional velocity and source distributions. However, only the distribution of the relative velocity on the lens plane is relevant to the microlensing rate, since it determines the lensing time scales $t_{\mathrm{E}}$ via the relation $t_{\mathrm{E}}=R_{\mathrm{E}} / v_{\mathrm{r}}$. As we show below, it is possible to analytically evaluate this distribution (Riffeser et al. 2006). Indeed, besides the velocity components along the los, both the remaining components of the source velocity can be analytically integrated. A final integration on the remaining component of the lens velocity is not possible, however, as a consequence of the assumed anisotropy of the velocity distribution.

The rationale of the evaluation is as follows. A first integration along the los for both sources and lenses leaves us with two Gaussian non diagonal distributions, that we project on the lens plane and diagonalise. This defines two frames on the lens plane whose axes, in general, will be misaligned. We then fix one of the two frames as a "reference", in particular that relative to the lens velocity distribution, we evaluate the relative velocity distribution by making use of Eq. (A.4) and integrate out the source velocity distribution components.

After integration along the line of sight the two-dimensional velocity distribution on the lens plane orthogonal to the los is

$f\left(\boldsymbol{v}_{(i) \mathrm{p}}\right) \mathrm{d}^{2} v_{(i) \mathrm{p}}=\frac{\mathrm{e}^{-\frac{v_{(i \mathrm{p}, 1}^{2}}{2 \sigma_{(i) \mathrm{p}, 1}^{2}}} \mathrm{e}^{-\frac{v_{(i) \mathrm{p}, 2}^{2}}{2 \sigma_{(i) \mathrm{p}, 2}^{2}}}}{(2 \pi)^{\frac{3}{2}} \sigma_{(i) \mathrm{p}, 1} \sigma_{(i) \mathrm{p}, 2}} \mathrm{~d}^{2} v_{(i) \mathrm{p}}$,

where the suffix $(i)$ indicate either lenses or sources. For the last component, because of the projection, $\boldsymbol{v}_{\mathrm{sp}}=x \boldsymbol{v}_{\mathrm{s}}$ and $\sigma_{\mathrm{sp}\{1,2\}}=$ $x \sigma_{\mathrm{s},\{1,2\}}$.

Let the principal axes of the intersection ellipse of the lens (source) proper velocity ellipsoid with the lens plane be $\left\{x_{l, 1}, x_{l, 2}\right\}$ $\left(\left\{x_{\mathrm{s}, 1}, x_{\mathrm{s}, 2}\right\}\right)$, hereafter we refer to the former frame as $O L, \omega$ the angle between $x_{1,1}$ and $x_{\mathrm{s}, 1}$ and $\left\{v_{\mathrm{spl}, 1}, v_{\mathrm{spl}, 2}\right\}$ the source velocity components in $O L$. $\omega$, as well as the values for the projected dispersion velocities, are fixed by the geometry, once the los has

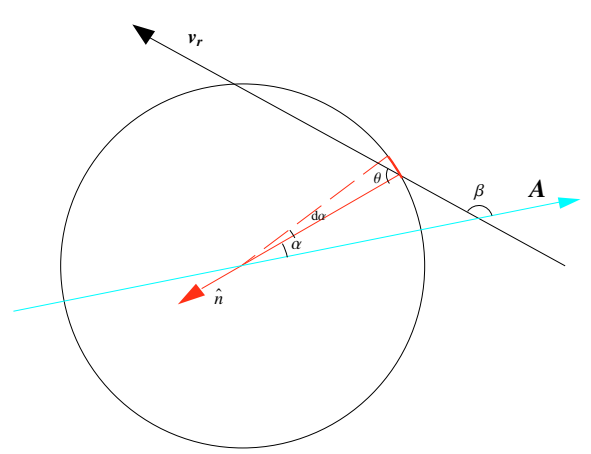

Fig. A.1. The microlensing tube cross section and the angles involved.

been chosen ( $\omega$ varies up to $\sim 8^{\circ}$, increasing with the Galactic latitude). The distribution for the relative velocity is then evaluated as

$$
\begin{aligned}
P\left(\boldsymbol{v}_{\mathrm{r}}\right) \mathrm{d}^{2} v_{\mathrm{r}} & =\int P\left(\boldsymbol{v}_{\mathrm{r}}, \boldsymbol{v}_{s p l}\right) \mathrm{d}^{2} v_{s p l} \mathrm{~d}^{2} v_{\mathrm{r}} \\
& =\int f\left(\boldsymbol{v}_{s p l}\right) f\left(\boldsymbol{v}_{l p}\right) \delta\left(\boldsymbol{v}_{\mathrm{r}}-\left(\boldsymbol{v}_{l p}-\boldsymbol{v}_{s p l}+\boldsymbol{A}\right)\right) \mathrm{d}^{2} v_{l p} \mathrm{~d}^{2} v_{s p l} \mathrm{~d}^{2} v_{\mathrm{r}} \\
& =\frac{1}{\pi \Sigma_{N}} \mathrm{e}^{-\left(\boldsymbol{v}_{\mathrm{r}}+\boldsymbol{A}\right) \cdot \Sigma \cdot\left(\boldsymbol{v}_{\mathrm{r}}+\boldsymbol{A}\right)} \mathrm{d}^{2} v_{\mathrm{r}}
\end{aligned}
$$

where

$$
\begin{aligned}
\Sigma= & \frac{1}{\Sigma_{N}^{2}}\left[\begin{array}{cc}
\Sigma_{a}+\Sigma_{b} & 0 \\
0 & \Sigma_{a}-\Sigma_{b}
\end{array}\right] \\
\Sigma_{a}= & \sigma_{l, 1}^{2}+\sigma_{l, 2}^{2}+x^{2}\left(\sigma_{s, 1}^{2}+\sigma_{s, 2}^{2}\right) \\
\Sigma_{b}= & \left(4 x^{2} \sin ^{2} \omega\left(\sigma_{l, 2}^{2}-\sigma_{l, 1}^{2}\right)\left(\sigma_{s, 1}^{2}-\sigma_{s, 2}^{2}\right)\right. \\
& \left.+\left(\sigma_{l, 1}^{2}-\sigma_{l, 2}^{2}+x^{2}\left(\sigma_{s, 1}^{2}-\sigma_{s, 2}^{2}\right)\right)^{2}\right)^{1 / 2} \\
\Sigma_{N}= & \left(\left(\sigma_{l, 1}^{2}+x^{2} \sigma_{s, 1}^{2}\right)\left(\sigma_{l, 2}^{2}+x^{2} \sigma_{s, 2}^{2}\right)\right. \\
& \left.+x^{2}\left(\sigma_{l, 1}^{2}-\sigma_{l, 2}^{2}\right)\left(\sigma_{s, 1}^{2}-\sigma_{s, 2}^{2}\right) \sin ^{2} \omega\right)^{1 / 2}
\end{aligned}
$$

Here both $\boldsymbol{v}_{\mathrm{r}}$ and $\boldsymbol{A}$ must be evaluated in $O L$. The angle between $\boldsymbol{A}$ and $x_{l, 1}$ is fixed by the geometry once we assign the los. Moreover, as Fig. A. 1 shows, $\beta=\pi+\alpha-\theta$ is the angle between $\boldsymbol{v}_{\mathrm{r}}$ and $\boldsymbol{A}$.

Finally, after moving to polar coordinates on the lens plane, $\left(v_{\mathrm{r}}, \theta\right)$, we reach the following expression for the microlensing rate

$$
\begin{aligned}
\mathrm{d} \Gamma= & 2 f(\mu) \rho_{\mathrm{l}} \frac{\rho_{\mathrm{s}} D_{\mathrm{s}}^{2} \mathrm{~d} D_{\mathrm{s}}}{\mathrm{I}_{\mathrm{s}}} R_{\mathrm{E}}\left(\mu, D_{\mathrm{l}}, D_{\mathrm{s}}\right) u_{\mathrm{t}} \\
& \times P\left(v_{\mathrm{r}}, \alpha\right) v_{\mathrm{r}}^{2} \mathrm{~d} v_{\mathrm{r}} \mathrm{d} \mu \mathrm{d} D_{\mathrm{l}} \mathrm{d} D_{\mathrm{s}} \mathrm{d} \alpha,
\end{aligned}
$$

where we have exploited the periodicity of the trigonometric functions involved in $P\left(v_{\mathrm{r}}\right)$ to analytically integrate over $\theta$ (this provides the factor " 2 "). Because of the assumed anisotropic velocity dispersions, the analytical integration over $\alpha$ is not possible and the dependence on this variable survives in the relative velocity distribution. The expression for the differential rate $\mathrm{d} \Gamma / \mathrm{d} t_{\mathrm{E}}$ is easily obtained from Eq. (A.8) using the relation $v_{\mathrm{r}}=R_{\mathrm{E}} / T_{\mathrm{E}}$.

In Eq. (A.8) we have introduced the dependence of the lens number distribution on the mass of the lens, $\mu$, with the usual "factorisation hypothesis" stating that the lens mass distribution is independent of the lens spatial distribution 
(De Rujula et al. 1991). $\rho_{1}$ is the lens spatial distribution, $f(\mu)$ the lens mass function that we normalise as follows

$$
\int_{\mu_{\min }}^{\mu_{\max }} f(\mu) \mu \mathrm{d} \mu=\frac{\rho_{0,1}}{M_{\odot}}
$$

where $\rho_{0,1}$ is the central density.

\section{References}

Alcock, C., Allsman, R. A., Alves, D. R., et al. 2000, ApJ, 542, 281

Allen, P. R., Koerner, D. W., Reid, I. N., \& Trilling, D. E. 2005, ApJ, 625, 385

Barlow, R. 1989, Statistics. A guide to the use of statistical methods in the physical sciences, The Manchester Physics Series (New York: Wiley)

Benjamin, R. A., Churchwell, E., Babler, B. L., et al. 2005, ApJ, 630, L149

Binney, J., \& Merrifield, M. 1998, Galactic astronomy (Princeton, NJ: Princeton University Press)

Bissantz, N., \& Gerhard, O. 2002, MNRAS, 330, 591

Bissantz, N., Englmaier, P., Binney, J., \& Gerhard, O. 1997, MNRAS, 289, 651

Bissantz, N., Englmaier, P., \& Gerhard, O. 2003, MNRAS, 340, 949

Bissantz, N., Debattista, V. P., \& Gerhard, O. 2004, ApJ, 601, L155

Blum, R. D. 1995, ApJ, 444, L89

Cabrera-Lavers, A., Hammersley, P. L., González-Fernández, C., et al. 2007, A\&A, 465, 825

Calchi Novati, S., Paulin-Henriksson, S., An, J., et al. 2005, A\&A, 443, 911

Calchi Novati, S., De Luca, F., Jetzer, P., \& Scarpetta, G. 2006, A\&A, 459, 407

Chabrier, G. 2003, PASP, 115, 763

de Jong, J. T. A., Widrow, L. M., Cseresnjes, P., et al. 2006, A\&A, 446, 855

De Rujula, A., Jetzer, P., \& Masso, E. 1991, MNRAS, 250, 348

Dehnen, W. 2000, AJ, 119, 800

Dehnen, W., \& Binney, J. 1998, MNRAS, 294, 429

Dwek, E., Arendt, R. G., Hauser, M. G., et al. 1995, ApJ, 445, 716

Evans, N. W., \& Belokurov, V. 2002, ApJ, 567, L119

Freudenreich, H. T. 1998, ApJ, 492, 495

Glicenstein, J.-F. 2003, ApJ, 584, 278

Gould, A. 2000, ApJ, 535, 928

Gould, A. 2003, ApJ, 583, 765

Grenacher, L., Jetzer, P., Strässle, M., \& de Paolis, F. 1999, A\&A, 351, 775

Griest, K. 1991, ApJ, 366, 412
Griest, K., Alcock, C., Axelrod, T. S., et al. 1991, ApJ, 372, L79

Gyuk, G. 1999, ApJ, 510, 205

Hamadache, C., Le Guillou, L., Tisserand, P., et al. 2006, A\&A, 454, 185

Han, C., \& Gould, A. 1995, ApJ, 447, 53

Han, C., \& Gould, A. 1996, ApJ, 467, 540

Han, C., \& Gould, A. 2003, ApJ, 592, 172

Holtzman, J. A., Watson, A. M., Baum, W. A., et al. 1998, AJ, 115, 1946

Jetzer, P. 1994, ApJ, 432, L43

Kent, S. M. 1992, ApJ, 387, 181

Kiraga, M., \& Paczynski, B. 1994, ApJ, 430, L101

Kozłowski, S., Woźniak, P. R., Mao, S., et al. 2006, MNRAS, 370, 435

Kroupa, P. 2002, Science, 295, 82

Kroupa, P. 2007, [arXiv: astro-ph/0703124]

Mancini, L., Calchi Novati, S., Jetzer, P., \& Scarpetta, G. 2004, A\&A, 427, 61

Minchev, I., Nordhaus, J., \& Quillen, A. C. 2007, ApJ, 664, L31

Paczyński, B. 1986, ApJ, 304, 1

Paczynski, B. 1991, ApJ, 371, L63

Paczynski, B., Stanek, K. Z., Udalski, A., et al. 1994, ApJ, 435, L113

Peale, S. J. 1998, ApJ, 509, 177

Picaud, S., \& Robin, A. C. 2004, A\&A, 428, 891

Popowski, P. 2001, in Microlensing 2000: A New Era of Microlensing Astrophysics, ed. J. W. Menzies \& P. D. Sackett (San Francisco: ASP), ASP Conf. Ser., 239, 244

Popowski, P., Griest, K., Thomas, C. L., et al. 2005, ApJ, 631, 879

Rattenbury, N. J., Mao, S., Debattista, V. P., et al. 2007a, MNRAS, 378, 1165

Rattenbury, N. J., Mao, S., Sumi, T., \& Smith, M. C. 2007b, MNRAS, 378, 1064

Rich, R. M., Reitzel, D. B., Howard, C. D., \& Zhao, H. 2007, ApJ, 658, L29

Riffeser, A., Fliri, J., Seitz, S., \& Bender, R. 2006, ApJS, 163, 225

Roulet, E., \& Mollerach, S. 1997, Phys. Rep., 279, 67

Sevenster, M., Saha, P., Valls-Gabaud, D., \& Fux, R. 1999, MNRAS, 307, 584

Smith, M. C., Wozniak, P., Mao, S., \& Sumi, T. 2007, MNRAS, 380, 805

Stanek, K. Z., Udalski, A., Szymanski, M., et al. 1997, ApJ, 477, 163

Sumi, T., Woźniak, P. R., Udalski, A., et al. 2006, ApJ, 636, 240

Tisserand, P., Le Guillou, L., Afonso, C., et al. 2007, A\&A, 469, 387

Vallenari, A., Pasetto, S., Bertelli, G., et al. 2006, A\&A, 451, 125

Wood, A. 2007, MNRAS, 380, 901

Wood, A., \& Mao, S. 2005, MNRAS, 362, 945

Zhao, H., \& Mao, S. 1996, MNRAS, 283, 1197

Zhao, H., Spergel, D. N., \& Rich, R. M. 1995, ApJ, 440, L13

Zhao, H., Rich, R. M., \& Spergel, D. N. 1996, MNRAS, 282, 175

Zoccali, M., Cassisi, S., Frogel, J. A., et al. 2000, ApJ, 530, 418 